\title{
Energy Attentive Pre-fault Detection Mechanism with Multilevel Transmission for Distributed Wireless Sensor Network
}

\author{
Roshani Talmale $^{1 *}$, M. Nirupama Bhat ${ }^{1}$, Nita Thakare ${ }^{2}$ \\ ${ }^{1}$ Vignan's University, Deemed to be University, Vadalamudi, AP, India \\ ${ }^{2}$ Priyadarshani College of Engineering, Nagpur, M.S., India
}

Corresponding Author Email: roshanikambe2011@gmail.com

https://doi.org/10.18280/ria.330203

Received: 10 January 2019

Accepted: 29 March 2019

\section{Keywords:}

wireless sensor network, pre fault

detection, routing, energy-efficiency

\begin{abstract}
To recognize the vision of ambient intelligence, wireless sensor networks (WSNs) are broadly used to invent real time and smart applications. Energy has been a major concern in sensor networks. Another challenging issue of wireless sensor network is to discover the imperfect node and recover them to maintain network topology. Enhancement of network lifetime, reliability and scalability also depends on fault detection and recovery mechanism. Distributed Energy Attentive Pre Fault Detection Mechanism is proposed in this work for pre fault detection and multipath routing. The novel idea behind this approach is to recognize the sensor fault in advanced and performed the improvement action based on notification parameter like acknowledgement not received, residual energy is below threshold value and hope count distance. It enables a predesor node to rapidly identify and efficiently categories the next energy aware shortest path, upon steering failure, rather than relying from the starting place. Extensive simulations are performed and result demonstrates that the proposed algorithms perform well on various performance indicator metrics such as throughput, energy consumption, packet drop ratio, and communication delay.
\end{abstract}

\section{INTRODUCTION}

Wireless sensor network has countless potential and gained enormous interest to develop all real time application. Sensor nodes are operational with a set of radio transceiver, transducer, a controller unit and memory unit using which they can acquire and process data from the deployed regions [1]. The sensor nodes can self structure in order to form a multihop network and forward the data to a Sink node. In WSN, each sensor node has restricted resources like less battery power, limited storage capacity, less bandwidth, since maintain network topology and increase network lifetime becomes a major challenge. Sensor nodes are mostly deployed in ruthless environments leads to higher probabilities of fault occurrence [2]. Sensor node faults are categorized in to static fault, gain fault, stuck at fault and transit fault. The fault which occurs due to bad calibration of sensing unity called as static fault. In a period of time if the change rate of sensed data is different to the expectation referred as gain fault. Stuck-at fault is occurred when there is no variation of sensed data series and transit fault is when sensed values are out of ranged of normal running [3]. In order to maintain the network in functioning situation at gainful level we need to consider the fault recovery management. Fault detection and recover the fault is another challenge. Wireless Sensor Networks are multi-path or multihop networks. In these networks, the transmission of the packet depends on the various intermediary nodes. These intermediary nodes are used to transmit the data packet to the destination. Previously, many researchers discussed the issues about sensor node like limited memory, lesser battery power, minimum computational timing and capacity etc. In spite of these limitation, wireless sensor network is used in almost all kind of application based on real time data. In proposed work our aim is to design an energy efficient routing path by detecting pre fault detection. The proposed work enables the network to be self managed and will face the challenge of fault tolerance in energy efficient manner. The proposed framework will increase the lifetime of sensor network also [4].

The most significant reason for WSN failure is deploying in hostile environment. Fault tolerance routing is about persistently maintaining an optimal performance, despite having faulty nodes. Energy consumption is another challenge in WSN. Using actuator wireless sensor actuator network (WSAN) energy issue of sensor network can be solved up to certain extent. Actuator node is having more computing capabilities and a longer battery life. Sensors node performed sensing and if any event is detected then forward the data to their close actuators. Actuator is responsible to take action on the events and informed the neighbored node about event happen. The application like real time monitoring, tracking of malicious objects in targeted region, security, identify the chemical attack, WSANs can potentially be used [5-6].

The number of routing algorithms has been designed during the past few decades. Many researchers have focused on overcoming various failures that affect the energy efficiency, network scalability of WSN. Energy saving routing protocol has been comprehensively studied to enhance the routing of data forwarding in WSN to choose the energy efficient shortest path and alternative paths in respond to node failure. Hakki Bagci et al., [9] introduced distributed fault detection algorithm known as disjoint path vector algorithm. The methodology is based on two layered architecture where lower layer consists of low energy sensor and upper layer hold high capacity sensor. Actuators are used to act on events. The main 
objective is to minimize the energy consumption which leads to minimize the fault. Md. Azaharuddin et al., [10] proposed fault model where permanent failure of $\mathrm{CH}$ is considered. The proposed model detects the fault if hardware problem occurs or in case of complete energy depletion of cluster head node. Rimpi Goyal et al., [11] proposed Fault detection mechanism has performed for both intra- cluster and inter-cluster levels. Failure within the cluster is identified by the cluster head by comparing the sensed data of that member node and its neighbor nodes while detection process for cluster head's failure is implemented by the detector. Ye Zhao et al. [12] integrated a distributed model for fault detection and topology accommodation. The proposed approach is based on distributed state filtering. Prasenjit Chanak et al. [13] presented FTMRS (Fault Tolerant Multipath Routing). Shortest path is used for main data routing and generates two backup paths in case of node failure.

Zidi et al., [14] proposed an algorithm based on support Vector Machine to define decision function. This decision function is executed and detect anomalous sensor. It also makes use of machine learning tool and semi-supervised method for recognition of faulty sensor nodes and deal with failures in WSNs. To augment the knowledge performance, the proposed method initiates a label propagation system which is based on local kernel density estimation. It calculates the posterior possibility of a scene that belongs to various malicious and faulty modes. In Ref. [15], author suggested fault detection and fault recovery scheme. This algorithm is based on two steps one is tree construction and identification of sleeping node. Whereas in other steps recovered the faulty node and update connection of sensor node network. In Ref. [16], the author proposed a new matrix calculus approach in which one parameter is consider i.e. round trip path row is generated. By using constructed RTP it is feasible to recognize several faulty nodes by evaluating row wise delay and column wise RTPs delay. Santoshinee et. al. [17] designed novel application which is used for fire forest monitoring. The author suggested a model which is used to identify faulty node and focus on fire monitoring.

In Ref. [18], author implemented (GA) genetic algorithm clustered based fault tolerant approach. Cluster head (CHs) are elected based on residual energy and energy efficient distance only. However, the sensing exposure region should be measured while selecting the $\mathrm{CH}$. Furthermore, the cluster associate faults are considered by a three-way handshake messages which leads to advanced operating cost. In Ref. [19], Mehdi et. al., tried to identify a distributed fault in WSN and presented a protocol to increased lifetime. The author suggested a mechanism to diagnose the hard failures in sensor nodes and applying counteract measures to make certain reliability and resiliency of the WSN. The parameter included separating malfunction nodes and topology modification.

Henna et al., proposed proficient disjoint coverage algorithm achieve energy efficiency and fault tolerance by preparing different disjoint set covers with minimum relay energy. On one hand, spate disjoint set achieve fault tolerance by providing double coverage [20]. Farnaz Pakdel et al., [21] proposed the strategy which balances in the density of the cluster cause to reschedule the finish time of the cluster head node and minimizes the collision because of less energy balance in clusters. The author proposed innovation based on fuzzy logic systems. Only cluster head fault can be detected and recover in proposed technique. Haiying Shen et al., [22] proposed fault tolerant algorithm based on Kautz graph method. It is profitable by allowing a node to directly calculate different routing paths from source to destination simply based on node IDs. In order to tackle the problem of node failure rate, multi-path routing in a Kautz cell is used. It avoids retransmission of lost data from source node, from previous intermediate node it retransmit to destination.

However, none of the above existing work addresses the pre fault detection and very few authors have suggested the routing techniques that address energy efficient and fault tolerant issues together. In proposed work we consider the pre fault detection in energy efficient manner. In proposed design we consider different types of Fault like permanent fault, transit fault and stuck in to fault in order to performed real time transmission and improve the performance of WSN. There are three fault diagnosis approach based on sensor node decision capacity like centralized, distributed and hybrid. Soft and hard node failure is broad categorization of sensor fault. Transmission failure, multipath fading, interference and permanent hardware failure are main causes of hard fault. It leads to high loss rate and delays in network communication. On the other hand sensor node communicates continuously but predicting faulty values cannot perform like secure routing node [7-8]. In this document, we have made the following major contributions.

- $\quad$ Proposed a novel approach for identifying the pre fault detection using the concept of Kuartz graph.

- Chosen the efficient routing path based on decision taken by actuator node and energy threshold value.

- In different scenario of WSN extensive experiments has been performed on the proposed algorithm and presented computational analysis.

- Simulation results are compared with existing algorithms and exhibit the development of the proposed mechanism.

The paper is divided in to the followed sections. Section two describes the network model and terminology. Section three describes the proposed algorithm and section four presents experimental results. Section five is about the conclusion of experimental analysis.

\section{NETWORK MODEL AND TERMINOLOGY}

In proposed network model we consider partial heterogeneous sensor network which consist of sensor node (S) and actuator nodes (A) with $\mathrm{S}<\mathrm{A}$. Sensor nodes and actuator are randomly deployed in to the targeted region. Each sensor node is having communication range Smax. Sensor nodes are joining to neighboured node if they are in communication range. Actuators are coordinating event if any activity will happen in network not in actual data transmission. The proposed model will focus on sensor to sensor and sensor to base station communication. All communication is using wireless link. We represent network topology with Kautz graph. It is having novel approach to resolve fault tolerant, means enables a network to continue operating properly in the event of the failure.

Following terminologies are used in proposed algorithm to design Kautz graph structure and calculate energy consumption, residual energy, distances and hop count value.

1. Sensor nodes are labelled as $\left\{s_{i}, s_{i+1}, s_{i+3} \ldots ., s_{N}\right\}$ where, $1 \leq i \leq N$, 
2. The set of Actuator node is denoted by $a=\{a 1, a 2, \ldots$, $a M\}$ and $B S$ indicates the base station .

3. Kautz graphs is directed graph, $K_{M}^{N+1}$ where $\mathrm{M}$ is degree and $\mathrm{N}+1$ is dimension.

$\mathrm{S}_{\mathrm{i}}$ belongs to the alphabet of $\mathrm{M}+1$ letter $\mathrm{A}=(0,1, \ldots \mathrm{M})$

The arc of the Kautz graph are $\left\{\left(s_{1}, s_{2} \ldots s_{N+1}, s_{2} s_{3} \ldots s_{N}\right.\right.$ $\left.s_{N+1}\right), S i \in A, S i \neq S i+1$ [22].

4. $E_{T x \text {-elec }}$ is the energy consumption for transmission electronics and $E_{T x-a m p}$ is the energy consumption to transmit amplifier.

The ratio of total energy consumed by sensor nodes while sending and receiving sensed information used radio model is same as discussed in Ref. [24]. In this model, based on actual connecting space among source and recipient, free space or multi-path channels are used. When the distance is less than an energy threshold value $(k O)$, then the free space (fsp) model is used, otherwise, the multipath $(\mathrm{Mp})$ model is used.

The Transmission energy ( $E_{T f s p}$ to transmit m-bit message $(m)$ to distance $(k)$ can be computed by Eq. (1) and Eq. (2) as given bellow.

$$
\mathrm{E}_{T f s p}(m, k)= \begin{cases}E_{T x \text {-elec }}+E_{T x \text {-amp }} k^{2} & k<k 0 \\ E_{T x \text {-elec }}+E_{T x \text {-amp }} k^{2} x, & k \geq k 0\end{cases}
$$

whereas, the computation power $\left(E_{R x}\right)$ required by sensor node to receive $(\mathrm{m})$ bit message is calculated by Eq. (3)

$$
E_{R x}(m)=E_{R x-e l e c} * m
$$

5. Residual energy for node is calculated by Eq. (4) as given below

$E n(r e s)=E i n t-\{(N t * E t-(N r * E r-(N i * E i-(N s * E s)\}$

where, En(res) denotes the energy residual of the sensor node, Eint represents energy initialization, $N t$ denotes the number of times data transmitted and Et represent transmitted energy, $\mathrm{Nr}$ denotes number of times node receives the data, $E r$ is the total energy required for received the data, and $N i$ and $N s$ represent number of times node is in ideal and sleep mode respectively. $E i$ is the energy in ideal mode and Es represent energy in sleep mode.

6. Dre $f$ is the distance between source node $S_{1}(x l, y l)$ and target node $S_{N}(x 2, y 2)$

It is calculated by Euclidian distance is computer by Eq. (5)

$$
\text { Dref }=\sqrt{(x 2-x 1)^{2}+(y 2-y 1)^{2}}
$$

12. HCount $(\mathrm{Si})$ calculates the number of hopes required to reach destination. If there is direct communication then Hcount( $\mathrm{Si}$ ) is one otherwise it is calculated as follows using Eq. (6)

$$
\text { Hcount }=\left\{\begin{array}{cc}
1, & \text { Direct } B S \\
1+\text { Hcount } & \text { Indirect } B S
\end{array}\right.
$$

\subsection{Efficient fault detection mechanism}

This section described the fault diagnosis mechanism using Kautz graph in sensor network. The Kautz graph topology supported the fault tolerant routing. There are various reasons for the failure of sensor node. Sensor node may fail due to battery problem, either permanent battery failure or not having sufficient battery capacity to operate. Most of the existing routing protocol only permanent battery failure fault is consider. In proposed routing mechanism two types of sensor fault is recognized. One is permanent fault due to battery depletion and another one identifies the node which is having low battery capacity. This is referred as pre fault detection. We use resource rich actuator between Kautz graph because they are able to exchange the information even though they are far way and responsible to detect the event happened in network and informed to neighbored sensor node in network. In the distributed scenario two actuators exchange their information for efficient routing. We first select one node as a source node and one as a destination node. Two nodes are connected by multi hoping. If there is direct connection from source to destination then hop count is 1 otherwise through recursive mechanism multi hop value is calculated. Two nodes are connected if distance is minimum than another path. The distance between two nodes is measured by Euclidian distance. In the beginning of each phase source node is sending message to neighbored node and wait for acknowledgement. If acknowledgment is received then only it calculates the distance otherwise ignore that node, otherwise permanent fault recognizes.

For pre fault detection, energy threshold value is set for all sensor node. While doing communication, if energy depletes below threshold value then it sends message to actuator with residual energy. Actuator informed other sensor node as well as other actuator also about faulty nodes. Once the information is received then source node is not considering that node in routing information. This mechanism helps to detect the faulty node in network which in turned to enhance the network lifetime.

\subsection{Energy aware topology maintenance}

In distributed large scale network low capacity sensor network is deployed in targeted region. To enhance the lifetime of network we have to apply topology maintenance strategy like duty cycle with awake and sleep period approach. It is not necessary to keep all sensors in active state and involved in data transmission process. In proposed DPFDRM the sensor node is active if energy is above threshold value and turned in to sleep node if it is having less energy i.e. below threshold value. The actuator checks the energy level of each node before calculating multiple routing paths. If any node is recognized as low energy level capacity then actuator informed the neighbored sensor node about the permanent sleep status and that node is not consider for routing. The actuator periodically exchanges the information with neighbored node to check the status of energy level. This mechanism is help to save the energy of each sensor node and prevent the fault in routing.

\subsection{Fault tolerant routing protocol}

In proposed Distributed Pre Fault Detection Routing Mechanism (DPFDRM), each sensor nodes are connected to other sensor node as per the smallest distance, degree and transmission range using Kautz graph topology. Actuators are involved to detect event in routing process. Kautz graph topology features support fault tolerance mechanism too. In case of sensor node failure, the proposed mechanism selects nest successor node to forward the data, then third possible shortest path and so on. As shown in figure 1, the available 
routing paths between source node $S_{1}$ and destination node $\mathrm{D}_{10}$, based on shortest path, residual energy and hope count are $1 \rightarrow 4 \rightarrow 7 \rightarrow 10 \quad, \quad 1 \rightarrow 2 \rightarrow 5 \rightarrow 9 \rightarrow 10$
$1 \rightarrow 2 \rightarrow 5 \rightarrow 8 \rightarrow 10 \quad$ and $\quad 1 \rightarrow 2 \rightarrow 3 \rightarrow 6 \rightarrow 7 \rightarrow 10 \quad$. In Figure 2 Source Node $S_{1}$ starts forwarding the data to node $D_{10}$ using the path $1 \rightarrow 2 \rightarrow 5 \rightarrow 9 \rightarrow 10$, but sensor node 9 is identified as a faulty node because acknowledgement (ACK) is not received or residual energy is less than threshold value. In such a case node 5 chooses the next successor node as per the parameter based on shortest path i.e. distance from source node to successor node $\left(\mathrm{D}_{\mathrm{SN}}\right)$ and distance from successor node to destination node $\left(\mathrm{D}_{\mathrm{ND}}\right)$ should be less than actual distance from source to destination (Dref). Second parameter is residual energy. By considering all the parameter the algorithm will select successor node is 8 instead of node 6 and final routing path is $1 \rightarrow 2 \rightarrow 5 \rightarrow 8 \rightarrow 10$. In proposed routing algorithm faulty nodes are pre detected and predesor node selects alternate path from itself to next node without creating any delay in transmission. However in previous Kautz based algorithm [22] pre fault detection issue is not consider hence more energy is required to retransmit the data from faulty node. To overcome this shortcoming, the proposed mechanism DPFDRM aims to designed a protocol, that can be recognized faulty node in advanced based on threshold energy value and acknowledge with low energy consumption. Figure 3 shows the flow chart of energy efficient pre fault detection routing algorithm.
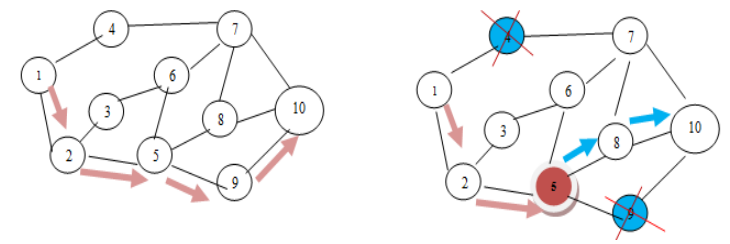

Figure 1. Node architecture

Figure 2. Fault tolerance

Pre fault detection is challenging task. Various approaches for finding the faults have been published. For getting the more efficient results, we have used the concept of Kautz graph.

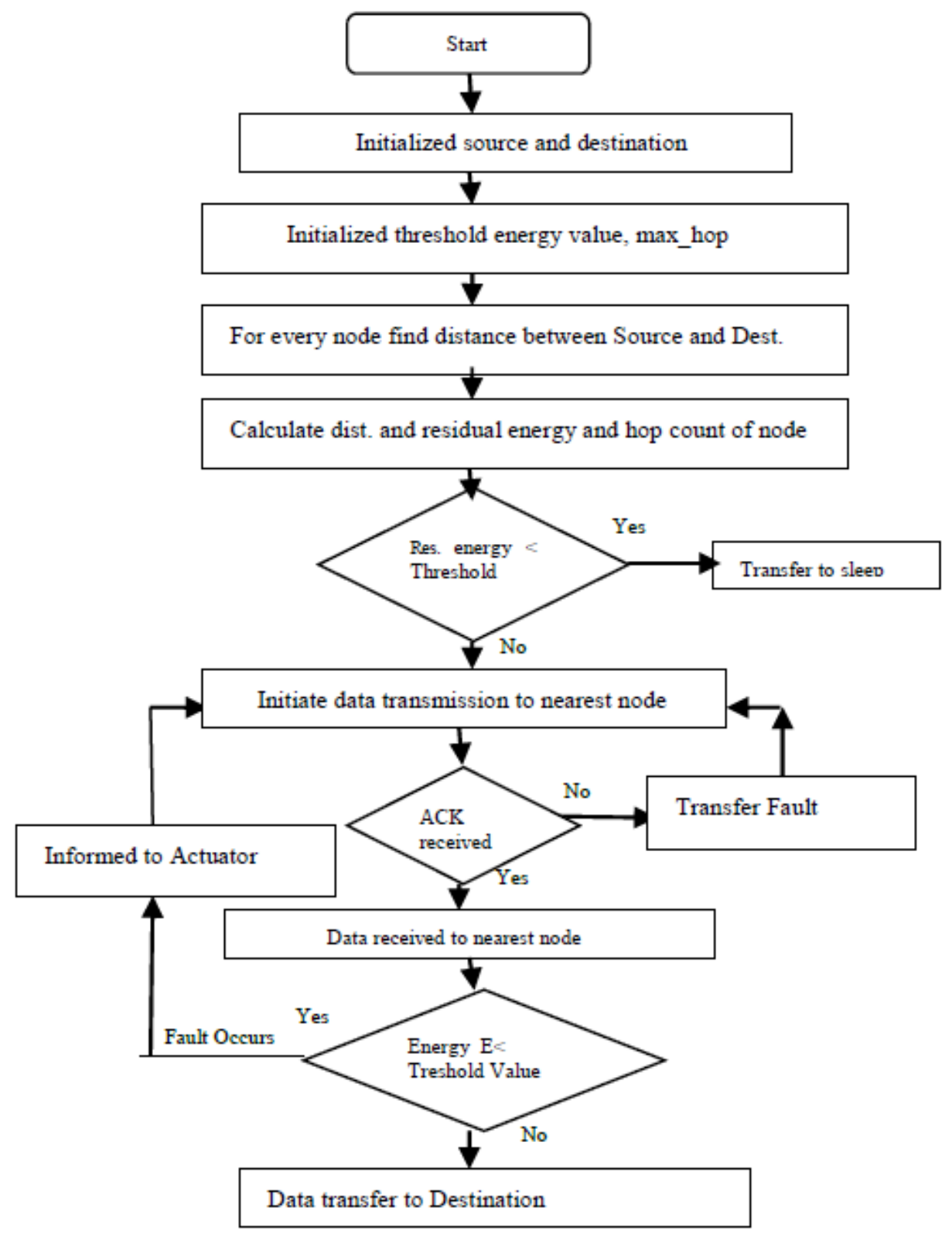

Figure 3. Flow chart for pre fault detection routing 


\section{PROPOSED ALGORITHM}

\subsection{Proposed algorithm}

\section{Energy Aware Pre Fault Detection Algorithm:}

Input: Source node, destination node, threshold value, residual energy

Output: Energy aware multipath routing

Steps:

Step 1: Select Source (S) and Destination (D)

Find Dref (distance between S and D)

Initialized maximum hop and traversal list

Step 2: /* Calculate residual energy of every node */

If Energy (e) $<$ Threshold energy then discard node

Else consider for routing data

Step 3: /* Execute for every node in the network */

Go to each node find distance from $\mathrm{S}$ to each node

Step 4: while start or receive data to forward do

Identify multiple paths to destination

Select minimum distance based on hop count and residual energy. Transmit the data to selected node

Step 4: If energy of selected node is above threshold then transmit data to successor node and wait for ACK

Step 5: If ACK received then proceed to find successor node Calculate the distance of successor node from source node to successor node $\left(\mathrm{D}_{\mathrm{SN}}\right)$ and from successor node to destination node $\left(\mathrm{D}_{\mathrm{NR}}\right)$

$\mathrm{D} 2=\mathrm{D}_{\mathrm{SN}}+\mathrm{D}_{\mathrm{NR}}$

Step 6: Performed routing only if

$D_{\text {SN }}<$ Dref

$\mathrm{D}_{\mathrm{NR}}<$ Dref

Step 7: Find minimum hops

If No. of hops is more than threshold then communicate directly from $\mathrm{S}$ to $\mathrm{D}$

Else follow the path.

Stop.

\section{EXPERIMENTAL RESULTS}

In this segment, we represented the simulation result observed by applying different set of parameter on sensor network for identifying pre faults and communication faults. Experimental results are carried out through the simulation run on NS2. In order to estimate the performance of proposed DPFDRM, conventional evaluation parameter metrics of WSN have been considered. The first performance evaluation parameter is residual energy of each node. Residual energy is remaining power with each node in the network. Second one is delay in communication. It is a normal latency from the event of data transmitted from source node to targeted station. Other parameter is packet drop ratio and communication energy consumption, i.e. Number of packets drop while transmission or receiving data and energy consumption and total energy dissipated of the network respectively. The table 1 shows parameters values which are used in simulation.

Table 2 depicts the simulation results with in different number of rounds. In simulation traditional parameter has set as shown in Table 1. For the comparison we used well known algorithm i.e. AOSD and REFER. Table 2 shows the comparison results in terms of delay, packet loss ratio and total energy consumption. It can be observed that DPFDRM performs better than the AOSD and REFER in terms of number of faulty nodes. In existing algorithm fault detection is not performed in advance. Retransmission is done in case of node failure and consumes more energy. In proposed algorithm in case of node failure, successor node immediately identify alternate shortest path with minimum hop count and without delay in transmission to destination.

Table 1. Simulation parameters

\begin{tabular}{|c|c|}
\hline Parameter & Value \\
\hline Sensor node & $30-50$ \\
\hline Actuator & 5 \\
\hline Energy Threshold & $200 \mathrm{~J}$ \\
\hline Packet Size & 1000 \\
\hline Initial Energy & $100 \mathrm{~J}$ \\
\hline Energy consumed in transmitter circuit & $50 \mathrm{nj} / \mathrm{bits}$ \\
\hline Energy consumed in amplifier & $10 \mathrm{pj} / \mathrm{bits}$ \\
\hline
\end{tabular}

Table 2. Simulation results

\begin{tabular}{|c|c|c|c|c|}
\hline $\begin{array}{c}\text { Algorith } \\
\text { m }\end{array}$ & $\begin{array}{c}\text { No of } \\
\text { Rounds }\end{array}$ & Delay(s) & $\begin{array}{c}\text { Packet } \\
\text { loss ratio }\end{array}$ & $\begin{array}{c}\text { Energy } \\
\text { Cons. (J) }\end{array}$ \\
\hline \multirow{4}{*}{ AOSD } & 100 & 0.001249 & 100.00 & 15.52 \\
\cline { 2 - 5 } & 200 & 0.001256 & 99.88 & 29.29 \\
\cline { 2 - 5 } & 300 & 0.001028 & 99.83 & 37.63 \\
\cline { 2 - 5 } & 400 & 0.001162 & 99.87 & 41.92 \\
\cline { 2 - 5 } & 500 & 0.001283 & 99.74 & 56.19 \\
\hline \multirow{4}{*}{ REFER } & 100 & 0.000414 & 98.84 & 74.93 \\
\cline { 2 - 5 } & 200 & 0.000314 & 99.30 & 84.38 \\
\cline { 2 - 5 } & 300 & 0.000652 & 98.86 & 73.12 \\
\cline { 2 - 5 } & 400 & 0.000432 & 98.47 & 53.01 \\
\cline { 2 - 5 } & 500 & 0.000322 & 98.48 & 73.54 \\
\cline { 2 - 5 } & 100 & 0.000246 & 98.16 & 8.98 \\
\cline { 2 - 5 } & 200 & 0.000307 & 97.88 & 15.18 \\
\cline { 2 - 5 } & 300 & 0.000278 & 98.21 & 12.88 \\
\cline { 2 - 5 } & 400 & 0.000154 & 97.92 & 32.57 \\
\cline { 2 - 5 } & 500 & 0.000296 & 97.19 & 53.64 \\
\hline
\end{tabular}

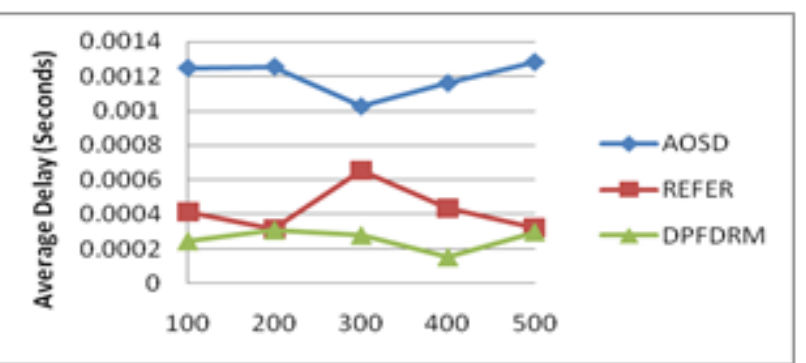

Figure 4. Average delay in different number of rounds

Figure 4 shows the average delay ratio from source node to destination in different number of communication. In proposed algorithm Distributed Pre Fault detection routing algorithm (DPFDRM) data delivery delay is very less as compared to AOSD and REFER. Figure 5 shows the packet delivery ratio from sender to receiver. In the proposed techniques, received number of packet percentage in base station with respect to source node data transmission is very high. From extensive simulation results are shows that in proposed Distributed Pre Fault Detection Routing Mechanism, performance of wireless sensor network is enhanced. This proposed mechanism improved the network performance of sensor network.

In DPFDRM, energy consumption rate of every node in different communication in case of node failure is shown in 
Figure 6. When faulty node percentage is less then ratio of energy dissipation of the sensor networks is high because maximum data delivery is happening to base station. In order to save the energy duty cycle approach is used in proposed algorithm.

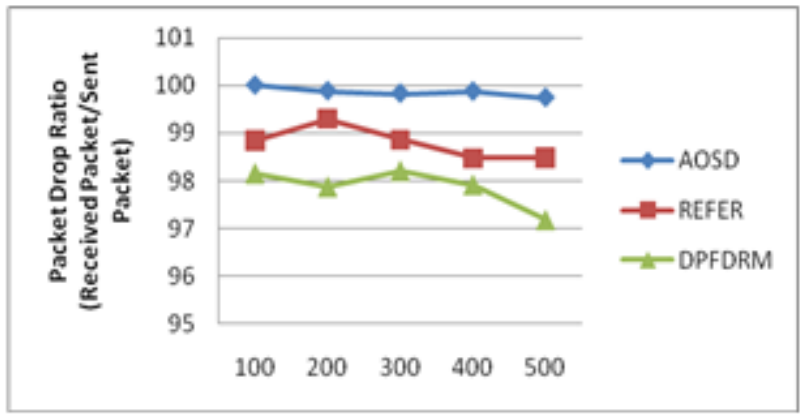

Figure 5. Packet delivery ratio in different scenario

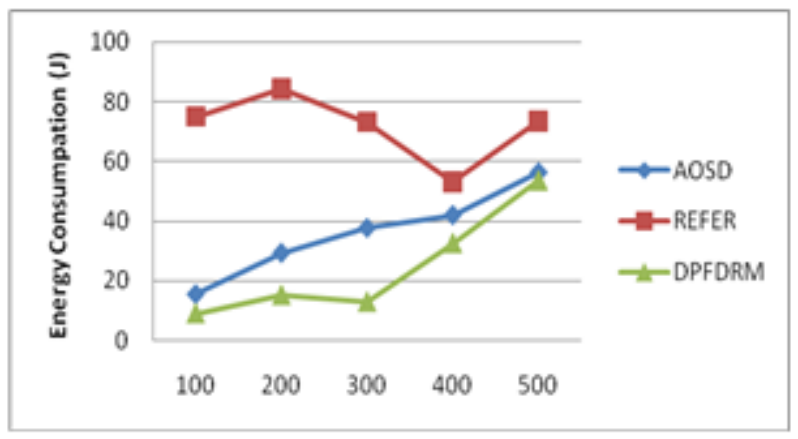

Figure 6. Average dissipated energy

\section{CONCLUSIONS}

The critical requirement of sensor network is to detect real time fault occurs in sensor node. Exiting routing protocol proposed by many researchers focuses on fault tolerance but very few have considered pre fault detection issue. In this paper, we studied exiting approach of fault detection and Qautz graph properties, shows optimal topology for WSN. In this paper we presented a distributed pre fault detection and recovery mechanism with multipath routing scheme for energy efficient wireless sensor and actuator Network. The proposed DPFDRM technique identifies the fault in advanced by considering residual energy and threshold value. It recovers node fault as well as transmission fault. It identifies multiple path for data transmission in energy efficient manner. In DPFDRM technique, fault understanding percentage is very high as compared to other existing techniques such as AOSD and REFER. Data forwarding time is very fast and energy aware even at high ratio of nodes failure. An extensive simulation output demonstrate the performance of DPFDRM as compared to AOSD, REFER routing techniques. The future scope is to enhance the quality of service and analyzes the complexity of the proposed algorithm for scalable network.

\section{REFERENCES}

[1] Al-Karaki, J.N., Kamal, A.E. (2004). Routing techniques in wireless sensor networks: A survey. IEEE Wireless
Communications,

11(6):

6-28.

https://doi.org/10.1109/MWC.2004.1368893

[2] Akyildiz, I.F., Su, W., Sankarasubramaniam, Y., Cayirci, E. (2002). Wireless sensor networks: A survey. Computer Networks, 38(4): 393-422. https://doi.org/10.1016/S1389-1286(01)00302-4

[3] Gao, Z.W., Cecati, C., Ding, S.X. (2015). A survey of fault diagnosis and fault-tolerant techniques-part I: Fault diagnosis with model-based and signal-based approaches. IEEE Transactions on Industrial Electronics, 62(6):

3757-3767. https://doi.org/10.1109/TIE.2015.2417501

[4] Zhang, Z.Y., Mehmood, A., Shu, L., Huo, Z.Q., Zhang, Y., Mukherjee, M. (2018). A survey on fault diagnosis in wireless sensor networks. IEEE Access, 6: 11349-11364. https://doi.org/10.1109/ACCESS.2018.2794519

[5] Guleria, K., Verma, A.K. (2018). Comprehensive review for energy efficient hierarchical routing protocols on wireless sensor networks. Wireless Networks, 25(3): 1159-1183. https://doi.org/10.1007/s11276-018-1696-1

[6] Karlof, C., Wagner, D. (2003). Secure routing in sensor networks: Attacks and countermeasures. Ad Hoc Networks, 1(2-3): 293-315. https://doi.org/10.1016/S1570-8705(03)00008-8

[7] Deng, J., Han, R., Mishra, S. (2006). INSENS: Intrusiontolerant routing in wireless sensor networks. Computer Communications in Dependable Wireless Sensor Networks, 29(2): 216-230. https://doi.org/10.1016/j.comcom.2005.05.018

[8] Ganesan, D., Govindan, R., Shenker, S., Estrin, D. (2001). Highly-resilient, energy-efficient multipath routing in wireless sensor networks. ACM SIGMOBILE Mobile Computing and Communications Review, 5(4): 11-25. https://doi.org/10.1145/509506.509514

[9] Bagci, H., Korpeoglu, I., Yazıc1, A. (2015). A distributed fault-tolerant topology control algorithm for heterogeneous wireless sensor networks. IEEE Transactions on Parallel and Distributed Systems, 26(4): 914-923. https://doi.org/10.1109/TPDS.2014.2316142

[10] Azharuddin, Md., Jana, P.K. (2015). A distributed algorithm for energy efficient and fault tolerant routing in wireless sensor networks. Springer Wireless Networks, 21(1): 251-267. https://doi.org/10.1007/s11276-0140782-2

[11] Goyal, R., Sran, S.S. (2016). Fault detection for the cluster-based system in wireless sensor networks. International Conference on Recent Cognizance in Wireless Communication \& Image Processing, pp. 719727. https://doi.org/10.1007/978-81-322-2638-3_80

[12] Zhao, Y., He, X., Zhou, D.H. (2017). Distributed fault source detection and topology accommodation design of wireless sensor networks. IECON 2017 - 43rd Annual Conference of the IEEE Industrial Electronics Society, pp $5529-5534$ https://doi.org/10.1109/IECON.2017.8216957

[13] Chanak, P., Samanta, T., Banerjee, I. (2013). Faulttolerant multipath routing scheme for energy efficient wireless sensor networks. International Journal of Wireless \& Mobile Networks (IJWMN), 5(2): 33-45. https://doi.org/10.5121/ijwmn.2013.5203

[14] Zidi, S., Moulahi, T., Alaya, B. (2018). Fault detection in wireless sensor networks through SVM classifier. IEEE Sensors Journal, 18(1): 340-347. https://doi.org/10.1109/JSEN.2017.2771226 
[15] Zhao, M,B., Tian, Z.Y., Chow, T.W.S. (2017). Fault diagnosis on wireless sensor network using the neighborhood kernel density estimation. Neural Computing and Applications, 31(8): 4019-4030. https://doi.org/10.1007/s00521-018-3342-3

[16] Palanikumar, R., Ramasamy, K. (2018). Effective failure nodes detection using matrix calculus algorithm in wireless sensor networks. Cluster Computing, 1-10. https://doi.org/10.1007/s10586-017-1566-0

[17] Mohapatr, S., Khilar, K.P. (2016). Forest fire monitoring and detection of faulty nodes using wireless sensor network 2016 IEEE Region 10 Conference (TENCON), pp. 3232-3236 https://doi.org/10.1109/TENCON.2016.7848647

[18] Rajeswari, K., Neduncheliyan, S. (2017). Genetic algorithm based fault tolerant clustering in wireless sensor network. IET Commun., 11(12): 1927-1932. https://doi.org/10.1049/iet-com.2016.1074

[19] Cheraghlou, M.N., Khadem-Zadeh, A., Haghparast, M. (2016). Increasing lifetime and fault tolerance capability in wireless sensor networks by providing a novel management framework. Wireless Personal Communications, $\quad$ 92(2): 603-622. https://doi.org/10.1007/s11277-016-3559-3

[20] Henna, S. (2017). Energy efficient fault tolerant coverage in wireless sensor networks. Hindawi Journal of Sensors,
2017: 1-11. https://doi.org/10.1155/2017/7090782

[21] Pakdel, F., Esmaeilpour, M. (2016). Fuzzy logic method for enhancement fault tolerant of cluster head in wireless sensor networks clustering. TEM Journal, 5(3): 268-276. https://doi.org/10.18421/TEM53-03

[22] Shen, H.Y., Li, Z. (2015). A Kautz-based wireless sensor and actuator network for real-time, fault-tolerant and energy-efficient transmission. IEEE Transactions on Mobile Computing, 15(1): 1-16. https://doi.org/10.1109/TMC.2015.2407391

[23] Elsayed, W.M., Sabbeh, S.F., Riad, A.M. (2017). A distributed fault tolerance mechanism for selfmaintenance of clusters in wireless sensor networks. Arabian Journal for Science and Engineering, 43(12): 6891-6907. https://doi.org/10.1007/s13369-017-2868-5

[24] Titouna, C., Ari, A.A.A., Moumen, H. (2018). FDRA: Fault detection and recovery algorithm for wireless sensor networks. Springer International Publishing AG, part of Springer Nature 2018 M. Younas et al. (Eds.): MobiWIS 2018, LNCS 10995, pp. 72-85. https://doi.org/10.1007/978-3-319-97163-6_7

[25] Heinzelman, W., Chandrakasan, A., Balakrishnan, H. (2000). Energy-efficient communication protocols for wireless micro sensor networks. In Proc. 33rd IEEE Hawaii International Conference on System Sciences, 8: 4-7. https://doi.org/10.1109/HICSS.2000.926982 